\title{
Prognostic relevance of rosette-like features in osteosarcoma
}

\author{
K Okada, T Hasegawa, R Yokoyama, Y Beppu, E ltoi
}

J Clin Pathol 2003;56:831-834

See end of article for authors' affiliations

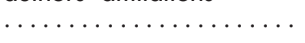

Correspondence to: Dr K Okada, MD

Department of Orthopaedic Surgery, Akita University School of Medicine, 1-1-1 Hondo, Akita 010-8543, Japan; cshokada@med.

akita-u.ac.jp

Accepted for publication 7 April 2003

\begin{abstract}
Aims: To clarify the prognostic relevance of rosette-like features and other clinicopathological and immunohistochemical variables in patients with osteosarcoma.

Methods: Clinicopathological and immunohistochemical variables were analysed in 131 patients with non-metastatic high grade conventional osteosarcoma, with particular attention to the prognostic impact of rosette-like features.

Results: Rosette-like features were present in 18 (14\%) cases. Rosette-like features were significantly associated with the osteoblastic subtype, numerous osteoclast-like giant cells, moderate pleomorphism, frequent haemangiopericytoma-like vascular patterns, epithelioid cytological features, positive immunoreactivity for epithelial membrane antigen and CD56, and negative staining for cytokeratin. In a multivariate analysis, rosette-like features (relative risk (RR), 3.8), a poor chemotherapy effect (RR, 2.9), and a tumour size of $10 \mathrm{~cm}$ or more (RR, 2.8) were identified as unfavourable prognostic factors.

Conclusions: Rosette-like features can easily be identified from routine histological slides and the relative risk in patients with non-metastatic, conventional osteosarcoma is as high as other well known prognostic factors, including large size and poor chemotherapy effect.
\end{abstract}

A s described previously, ${ }^{12}$ few osteosarcomas have rosette-like features with the production of osteoid in the centre. In a previous study, we reported that rosette-like features are an adverse factor for disease related mortality in patients with osteoblastic osteosarcoma. ${ }^{3}$ However, the relation between this histological feature and prognosis in all types of osteosarcoma is still obscure. The aim of our study was to clarify the prognostic relevance of rosettelike features and other clinicopathological parameters in patients with non-metastatic, high grade, conventional osteosarcoma.

\section{MATERIALS AND METHODS}

We reviewed more than 200 cases of osteosarcoma of the extremities or axial bones treated at the National Cancer Centre in Tokyo and the Akita University Hospital in Akita, Japan from 1962 to 1999. Clinical details and follow up information were obtained by reviewing medical charts. Microscope slides of the biopsy and the surgical specimen were reviewed. Patients with osteosarcoma in the jaw bones or cranium, low grade and high grade surface variants, dedifferentiated chondrosarcoma, primary metastasis at presentation, and secondary osteosarcomas were discarded from our study. Consequently, 131 patients with nonmetastatic, conventional osteosarcoma were studied. Sixteen patients with rosette-like features reported in our former study were included in our current study. ${ }^{3}$ All protocols were accepted by the review boards of our institutions. Informed consent was required from all patients or their legal guardians.

We evaluated the numbers of osteoclast-like giant cells, intensity of pleomorphism, frequency of the haemangiopericytoma-like pattern, and the epithelioid features of the tumour cells along with the rosette-like features using the haematoxylin and eosin stained slides of the biopsies. Preoperative chemotherapy was given and surgery was performed in 92 patients. In 87 of these 92 patients, the effect of chemotherapy was evaluated in the surgical specimen by assessing the percentage of necrosis of the tumour as follows: good, $\geqslant 95$; moderate, $\geqslant 90$; and poor, $<90$, but surgical specimens were not available in five patients. In the
87 patients, 50 patients were treated with combination chemotherapy, mainly of high dose methotrexate, doxorubicin, and cisplatin, with or without ifosfamide. The other 37 patients were treated with combination chemotherapy of high dose methotrexate, doxorubicin, and bleomycin; cyclophosphamide and dactinomycin were used in varying combinations.

In all the 131 cases, section of $4 \mu \mathrm{m}$ thickness were cut from the representative blocks of formalin fixed, paraffin wax embedded biopsy tissue. The sections were examined by the labelled streptavidin-biotin method, with the appropriate use of negative controls throughout, after pretreatment with heat induced epitope unmasking in $10 \mathrm{mM}$ citrate buffer, $\mathrm{pH}$ 6.0, in an autoclave at $121^{\circ} \mathrm{C}$ for 10 minutes. The primary antibodies were applied as follows: Ki-67 (clone MIB-1; 1/100 dilution; Immunotech, Marseilles, France), cytokeratin (clone AE 1/3; 1/100 dilution; Dako, Glostrup, Denmark), CD99 (clone O-13; 1/50 dilution; Signet, Dedham, Massachusetts, USA), epithelial membrane antigen (EMA; clone E29; 1/100 dilution, Dako), and CD56 (clone N-CAM; NCC-Lu-243; 1/200 dilution; Nihonkayaku, Tokyo, Japan). The MIB-1 labelling index was estimated as a continuous variable by counting the percentage of positive cell nuclei for each 100 tumour cells in the region of the tumour with the greatest density of staining. When evaluating the immunostaining for AEl/3, CD99, EMA, and CD56, only homogeneous staining was assessed as 2+ positive, and heterogeneous or focal staining was assessed as $1+$.

The following clinicopathological variables were subjected to analysis for their prognostic value by the Kaplan-Meier method, and the differences were compared by the log rank test: patient age; patient sex; tumour site; tumour size; surgical margin; tumour recurrence; chemotherapy; chemotherapy effect; histological subtype; Broder's grade; presence of osteoclast-like giant cells, pleomorphism, haemangiopericytoma-like pattern, epithelioid features, and rosette-like features; immunostaining for EMA, cytokeratin,

Abbreviations: $E M A$, epithelial membrane antigen; $R R$, relative risk 
CD56, and CD99; and MIB-1 labelling index. In the assessment of EMA, cytokeratin, CD56, and CD99, 2+ and $1+$ positive was categorised as positive, and negative staining as negative. The relative risk of each variable was estimated by Cox's proportional hazards model in univariate and multivariate analyses. Multivariate analysis was conducted with variable selection by a stepwise forward procedure. A $\chi^{2}$ test was used to evaluate the relation between rosette-like features and the other variables, including patient age; patient sex; tumour site; tumour size; chemotherapy effect; histological subtype; Broder's grade; presence of osteoclastlike giant cells, pleomorphism, haemangiopericytoma-like pattern, and epithelioid features; immunostaining for EMA, cytokeratin, CD56, and CD99; and MIB-1 labelling index. Differences at $\mathrm{p}<0.05$ were considered significant.

\section{RESULTS}

Histologically, a rosette-like feature with lace-like osteoid deposits in the centre was present in 18 tumours. In these areas, a small multinodular growth pattern between dilated blood vessels was prominent at low power (figs 1,2 ). Table 1 summarises the results of the univariate analysis. Follow up information on all 131 patients was available and covered periods ranging from 3 to 351 months (mean, 57 months). The following four variables were found to be adverse factors for overall survival: tumour size of $10 \mathrm{~cm}$ or more, inadequate surgical margin, rosette-like features, and poor chemotherapy effect. In a multivariate analysis, rosette-like features, poor chemotherapy effect, and tumour size of $10 \mathrm{~cm}$ or more were identified as unfavourable prognostic factors (table 2). Kaplan-Meier survival curves were plotted for patients according to the presence or absence of rosette-like features (fig 3).

In the $\chi^{2}$ test, rosette-like features significantly correlated with osteoblastic subtype, numerous osteoclast-like giant cells, moderate pleomorphism, frequent haemangiopericytoma-like pattern, epithelioid features, positive immunoreactivity for EMA and $\mathrm{CD} 56$, and negative staining for cytokeratin, but not with the other variables (table 3 ).

\section{DISCUSSION}

The prognostic factors of osteosarcoma, including axial tumour site, ${ }^{4}$ proximal tumour site, ${ }^{56}$ large tumour size, ${ }^{47}$ high alkaline phosphatase concentration, ${ }^{58}$ poor chemotherapy effect, ${ }^{49}{ }^{10}$ an inadequate surgical margin, ${ }^{24}{ }^{6}$ histological grade, ${ }^{1}$ spontaneous necrosis, ${ }^{11} \mathrm{p}$ glycoprotein, ${ }^{12}$ proliferating marker, ${ }^{13}{ }^{14}$ p53 expression, ${ }^{15}$ and HER-2/erb-B2 expression ${ }^{16}$ have already been reported. The current analysis showed that tumour size of $10 \mathrm{~cm}$ or more and histological rosette-like features in the biopsy were prognostic factors detectable at

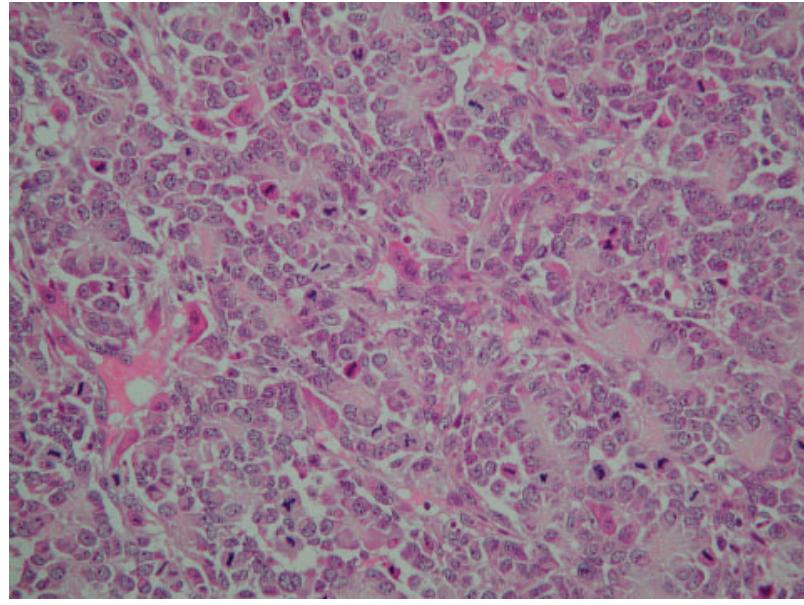

Figure 1 Rosette-like features of osteosarcoma (original magnification, $\times 200$ ).

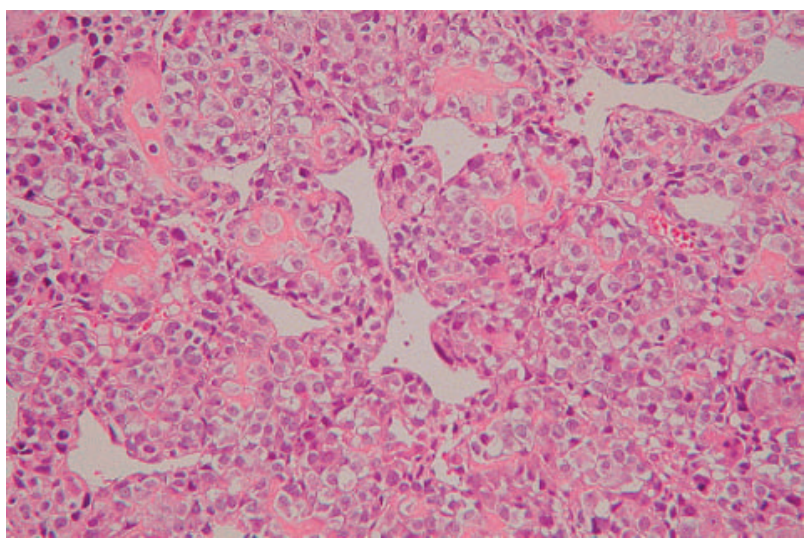

Figure 2 Low power view of a rosette forming epithelioid osteosarcoma shows a prominent haemangiopericytoma-like vascular pattern (original magnification, $\times 100)$.

diagnosis. A poor chemotherapy effect was also a prognostic factor, although an evaluation can only be made later in the disease course.

The relation between prognosis and the effect of neoadjuvant chemotherapy, as assessed by histological changes, has been well documented, and is now used universally in clinical practice. ${ }^{45910}$ Our current study showed that the existence of rosette-like features did not correlate with the

Table 1 Patient and tumour characteristics and univariate analysis for overall survival in 131 cases of high grade osteosarcoma

\begin{tabular}{|c|c|c|c|c|}
\hline Variable & No. of cases & $\% 5$ year survival rate $(95 \% \mathrm{Cl})$ & Log rank $p$ value & Relative risk $(95 \% \mathrm{Cl})$ \\
\hline Size $(\mathrm{cm})$ & & & 0.003 & \\
\hline$<10$ & 64 & 54.8 (41.1 to 68.4$)$ & & 1 \\
\hline$\geqslant 10$ & 67 & $28.6(17.2$ to 40.1$)$ & & 2.1 (1.3 to 3.4$)$ \\
\hline Surgical margin & & & $<0.001$ & \\
\hline Adequate & 112 & 48.5 (38.5 to 58.5 ) & & 1 \\
\hline Inadequate & 19 & $15.8(0$ to 32.2$)$ & & $2.9(1.7$ to 5.1$)$ \\
\hline Chemotherapy effect & & & 0.03 & \\
\hline Good & 20 & 55.2 (29.1 to 81.2$)$ & & 1 \\
\hline Moderate & 31 & 45.6 (22.6 to 68.6$)$ & & 1.2 (0.5 to 3.2$)$ \\
\hline Poor & 36 & 27.7 (12.5 to 43.0$)$ & & $2.6(1.1$ to 6.0$)$ \\
\hline Rosette-like feature & & & 0.008 & \\
\hline None & 113 & 45.8 (35.9 to 55.7 ) & & 1 \\
\hline Present & 18 & 13.5 (0 to 30.8 ) & & $2.2(1.2$ to 3.9$)$ \\
\hline
\end{tabular}


Table 2 Multivariate analysis for overall survival in 131 osteosarcomas

\begin{tabular}{llll}
\hline Variables & $\mathbf{p ~ V a l u e}$ & RR risk & $\mathbf{9 5 \%} \mathbf{C l}$ \\
\hline Size $(\geqslant 10 \mathrm{~cm})$ & 0.003 & 2.8 & 1.4 to 5.6 \\
Poor chemotherapy effect & 0.02 & 2.9 & 1.2 to 6.9 \\
Rosette-like features & 0.008 & 3.8 & 1.5 to 9.3 \\
\hline Cl, confidence interval; RR, relative risk. &
\end{tabular}

effect of chemotherapy (table 3), suggesting that tumours with this feature have an aggressive clinical behaviour, even when neoadjuvant chemotherapy is effective, as evaluated by conventional histological examination.

In a former analysis, we found that 16 cases, accounting for $5.7 \%$ of all osteoblastic osteosarcomas in long tubular bones of the extremities, showed rosette-like features in the biopsy. ${ }^{3}$ In all of these 16 tumours, a small multinodular growth pattern between dilated blood vessels showing a haemangiopericytoma-like appearance was prominent at low power. Osteoclast-like multinucleated giant cells were frequently seen within the nodules, and nuclear pleomorphism was not evident. In our current study, these morphological features were significantly correlated with the presence of rosette-like features. Rosette-like features in osteosarcomas can be seen in a gland-like arrangement, ${ }^{12}$ or as an epithelioid osteosarcoma with a nesting pattern. ${ }^{17}$ Thus, this histological variant could be viewed as a rare type of highgrade epithelioid appearing osteosarcoma.

"Our current study showed that the existence of rosettelike features did not correlate with the effect of chemotherapy (table 3), suggesting that tumours with this feature have an aggressive clinical behaviour, even when neoadjuvant chemotherapy is effective"

It is well known that epithelioid appearing osteosarcomas occasionally show positive immunoreactivity for cytokeratin, and their clinical course is similar to that of conventional

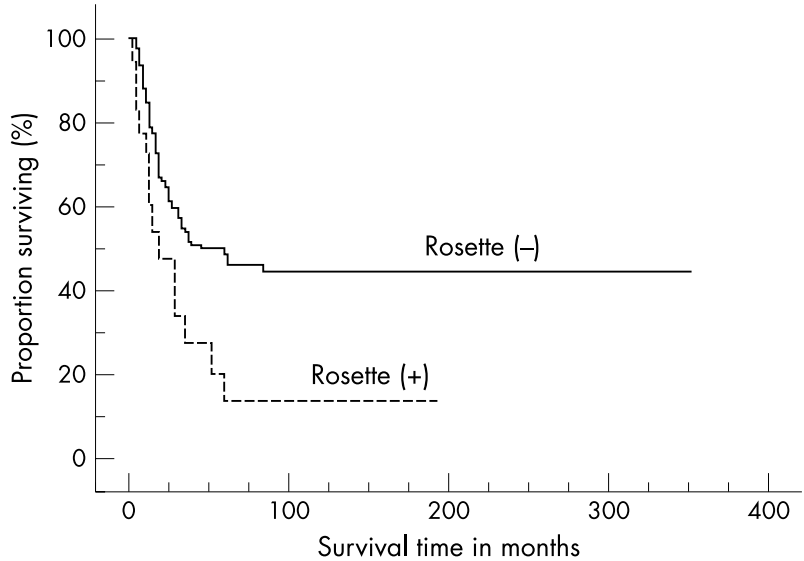

Figure 3 Kaplan-Meier survival curves of the patients with osteosarcoma according to the presence or absence of rosette-like features.

osteosarcomas. ${ }^{18}$ In contrast, osteosarcomas with rosette-like features, often associated with a positive reaction for EMA but absence of cytokeratin expression, displayed an aggressive clinical course. Expression of CD56 and CD99, seen in one third and one half of the osteosarcomas, respectively, had no prognostic impact. High proliferative activity, which was estimated to be an adverse prognostic factor in a previous study including low grade osteosarcomas, ${ }^{14}$ was not associated with prognosis in our current study. Similarly, the former analysis showed that the expression of MIB-1 in osteosarcomas with rosette-like features was not significantly different from that of osteoblastic osteosarcoma without rosette-like features. ${ }^{3}$ Proliferative activity may not be correlated with prognosis in patients with high grade osteosarcoma.

In conclusion, the rosette-like configuration can be easily identified in routine histological slides without the need to use either a molecular or a chromosomal approach, and its relative risk in patients with non-metastatic, conventional osteosarcoma is as high as other well known prognostic

\begin{tabular}{|c|c|c|c|c|}
\hline \multirow[b]{2}{*}{ Variable } & \multirow[b]{2}{*}{ No. of cases } & \multicolumn{2}{|c|}{ Rosette-like features } & \multirow[b]{2}{*}{$\mathrm{p}$ Value } \\
\hline & & None & Present & \\
\hline Histological subtypes & & & & 0.04 \\
\hline Osteoblastic & 100 & 82 & 18 & \\
\hline Chondroblastic & 14 & 14 & 0 & \\
\hline Fibroblastic & 17 & 17 & 0 & \\
\hline Osteoclast-like giant cell & & & & 0.006 \\
\hline Sparse & 114 & 102 & 12 & \\
\hline Numerous & 17 & 11 & 6 & \\
\hline Pleomorphism & & & & 0.007 \\
\hline Moderate & 87 & 70 & 17 & \\
\hline Pronounced & 44 & 43 & 1 & \\
\hline Haemangiopericytoma-like pattern & & & & $<0.001$ \\
\hline Infrequent & 60 & 59 & 1 & \\
\hline Frequent & 71 & 54 & 17 & \\
\hline Epithelioid feature & & & & 0.02 \\
\hline Not prominent & 90 & 82 & 8 & \\
\hline Prominent & 41 & 31 & 10 & \\
\hline Epithelial membrane antigen & & & & $<0.001$ \\
\hline Negative & 110 & 100 & 10 & \\
\hline Positive & 21 & 13 & 8 & \\
\hline Cytokeratin & & & & 0.02 \\
\hline Negative & 104 & 86 & 18 & \\
\hline Positive & 27 & 27 & 0 & \\
\hline CD56 & & & & $<0.001$ \\
\hline Negative & 89 & 87 & 2 & \\
\hline Positive & 42 & 26 & 16 & \\
\hline
\end{tabular}




\section{Take home messages}

- Rosette-like features can easily be identified from routine haematoxylin and eosin stained slides

- The relative risk of rosette-like features in patients with non-metastatic, conventional osteosarcoma is as high as other well known prognostic factors, including large size and poor chemotherapy effect

- Therefore, the presence of rosette-like features is useful for identifying those patients with osteosarcoma who have a poor prognosis

factors. Because widely used therapeutic courses have proved to be not effective, more extensive or alternative therapeutic courses may be required to eradicate this subtype of osteosarcoma.

\section{Authors' affiliations}

K Okada, E Itoi, Department of Orthopaedic Surgery, Akita University School of Medicine, Akita 010-8543, Japan

T Hasegawa, Pathology Division, National Cancer Centre Research Institute, Tokyo 104-0045, Japan

R Yokoyama, Y Beppu, Orthopaedic Divisions, National Cancer Centre Research Institute and Hospital, Tokyo 104-0045, Japan

\section{REFERENCES}

1 Unni KK. General aspects and data on 11,087 cases. In: Dahlin's bone tumours, 5th ed. Philadelphia: Lippincott-Raven, 1996:143-60.

2 Nakajima H, Sim FH, Bond JR, et al. Small cell osteosarcoma of bone. Review of 72 cases. Cancer 1997;79:2095-106.
3 Okada K, Hasegawa T, Yokoyama R. Rosette-forming epithelioid osteosarcoma. A histologic subtype with highly aggressive clinical behavior. Hum Pathol 2001;32:726-33.

4 Bielack SS, Kempf-Bielack B, Delling B, et al. Prognostic factors in high-grade osteosarcoma of the extremities or trunk: an analysis of 1,702 patients treated on neoadjuvant cooperative osteosarcoma study group protocols. J Clin Oncol 2002;20:776-90.

5 Provisor AJ, Ettinger $\amalg$, Nachman JB, et al. Treatment of nonmetastatic osteosarcoma of the extremity with preoperative and postoperative chemotherapy: a report from the Children's Cancer Group. J Clin Oncol 1997; 15:79-84.

6 Szendroi M, Papai Z, Koos R, et al. Limb-saving surgery, survival, and prognostic factors for osteosarcoma: the Hungarian experience. J Surg Oncol 2000;73:87-94.

7 Bieling $P$, Rehan N, Winkler $P$, et al. Tumor size and prognosis in aggressively treated osteosarcoma. J Clin Oncol 1996;14:848-58.

8 Bacci G, Picci P, Ferrari S, et al. Prognostic significance of serum alkaline phosphatase measurements in patients with osteosarcoma treated with adjuvant or neoadjuvant chemotherapy. Cancer 1993;71:1224-30.

9 Raymond AK, Chawla SP, Carrasco H, et al. Osteosarcoma chemotherapy effect: a prognostic factor. Semin Diagn Pathol 1987;4:212-36.

10 Rytting M, Pearson P, Raymond AK, et al. Osteosarcoma in preadolescent patients. Clin Orthop 2000:373:39-50.

11 Biörnsson J, Inwards CY, Wold LE, et al. Prognostic significance of spontaneous tumour necrosis in osteosarcoma. Virchows Arch A Pathol Anat Histopathol 1993;423:195-9.

12 Baldini N, Scotlandi K, Barbanti-Brodano G, et al. Expression of $\mathrm{P}$-glycoprotein in high-grade osteosarcoma in relation to clinical outcome. N Engl J Med 1995;333:1380-5.

13 Scotlandi K, Serra M, Manara MC, et al. Clinical relevance of Ki-67 expression in bone tumors. Cancer 1995;75:806-14.

14 Hasegawa T, Hirose T, Seki K, et al. Histological and immunohistochemical diversities, and proliferative activity and grading in osteosarcomas. Cancer Detect Prev 1997;21:280-7.

15 Toguchida J, Yamaguchi T, Ritchie B, et al. Mutation spectrum of the p53 gene in bone and soft tissue sarcoma. Cancer Res 1992:52:6194-9.

16 Gorlick R, Huvos AG, Heller G, et al. Expression of HER2/erbB-2 correlates with survival in osteosarcoma. J Clin Oncol 1999;17:2781-8.

17 Mirra JM, Gold RH, Picci P. Osseous tumours of intramedullary origin. In: Bone tumors. Clinical, radiologic, and pathologic correlations, Vol. 1. Philadelphia: Lea and Febiger, 1989:143-438.

18 Hasegawa T, Hirose T, Kudo E, et al. Immunophenotypic heterogeneity in osteosarcomas. Hum Pathol 1991;22:583-90.

\section{$\mathrm{ECHO}$}

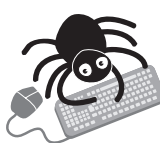

Please visit the Journal of Clinical Pathology website [www. jclinpath.com] for a link to the full text of this article.

\section{Chromosome 4 hyperploidy occurs early in premalignant Barrett's oesophagus}

G enetic aberrations are widespread in Barrett's oesophagus, with aneuploidy developing well before dysplasia can be identified.

Endoscopic cytology brushings from patients with oesophageal dysplasia, Barrett's metaplasia, and adenocarcinoma were cytospun to generate a single layer of interphase cells. The slides were prepared for FISH and examined with chromosome enumeration probes for the centromeres of chromosomes 4,8,20 and locus specific identifier probes for p53, 13q14 and 9p21/CEP9.

Chromosome 4 hyperploidy proved the commonest alteration, present in $89 \%$ of Barrett's patients, persisting in low grade dysplasia (LGD) and high grade dysplasia (HGD). The proportion of cells displaying this hyperploidy increased with neoplastic progression.

Chromosome 8 hyperploidy was also prominent being present in $71 \%$ of metaplastic Barrett's patients, 75\% of those with LGD and all those with HGD and adenocarcinoma.

Both losses and gains in chromosome 20 were detected. Only four of 20 men with metaplasia had lost chromosome Y but this increased to 38\% in LGD, 71\% in HGD, and $100 \%$ in adenocarcinoma.

The study has identified genetic instability in Barrett's metaplastic tissues, predominantly of chromosomes 4 and 8. This amplification may point to key genes on the chromosomes being mechanistically involved in initiation and progression of the lesion. Additionally, brush cytology coupled to FISH analysis is a suitable technique to identify early genetic abnormalities.

\ Gut 2003;52:623-628. 